\title{
La estrategia del debate en las competencias comunicativas orales en niños de la Institución Educativa Inicial № 318 Jean Piaget, Ucayali
}

\author{
The strategy of the debate in oral communication skills in children of the \\ Initial Education Institution N 0318 Jean Piaget, Ucayali
}

\author{
Atanacia Santacruz Espinoza ${ }^{1 *}$, Humberto Montenegro Muguerza ${ }^{2}$ \\ ${ }^{1}$ Universidad Nacional Intercultural de la Amazonia, Pucallpa, ${ }^{2}$ Universidad Nacional Hermilio Valdizán, Huánuco
}

\section{RESUMEN}

El objetivo fue demostrar los efectos de la estrategia del debate en las competencias comunicativas orales en los niños de 5 años de la Institución Educativa Inicial $N^{\circ} 318$ Jean Piaget - Yarinacocha, Ucayali al 2015. El estudio corresponde a una investigación cuantitativa en una muestra de 26 niños y niñas de 5 años de edad. Para la medición de la atención se utilizó una ficha de observación; la misma que se validaron y confiabilizaron. Los datos fueron procesados a través de los estadígrafos descriptivos y la $\dagger$ de Student, 0,05 de significancia y una (tc = $34,448>1,7081$ ). En los resultados se tiene que en el pretest el $100 \%$ de los niños se encontraba con una valoración entre 0 a 10; en el postest el $96 \%$ de los niños se ubicó con una valoración entre 11 a 13 y un $4 \%$ con una valoración de 14 a 17 o tuvo un logro previsto. La † calculada (tc $=34,448$ ) con 25 grados de libertad es mayor que la $t$ tabulada 0 crítica ( $t=1,7081)$ y cae en la zona de rechazo de la hipótesis nula, así también el valor del nivel crítico $(=0,000)$ es menor que el error estimado $(=0,05)$ entonces, se rechaza la hipótesis nula y se acepta la hipótesis de investigación, es decir, se concluye que la estrategia del debate ha producido efectos positivos en las competencias comunicativas orales en los niños de 5 años de la Institución Educativa Inicial $N^{\circ} 318$ Jean Piaget.

Palabras claves: Estrategia del debate, competencias comunicativas orales, lingüístico, pragmática, paralenguaje, kinésica.

\begin{abstract}
The objective was to demonstrate the effects of the debate strategy on oral communication skills in children of 5 years of the Initial Educational Institution №318 Jean Piaget - Yarinacocha, Ucayali to 2015. The study corresponds to a quantitative investigation in a simple of 26 boys and girls of 5 years of age. Fort he measurement of attention, an observation card was used; the same one that was validated and trusted. The data were proccessed through the descriptive statistics and Student's T, 0,05 of significance and one (tc $=34,448>$ 1.7081 ). In the results $100 \%$ of the children had a score between 0 and 10 in the pretest; in the posttest $96 \%$ of the children had a score between 11 to 13 and $4 \%$ with a score of 14 to 17 or had an expected accomplishment. The calculated $t$ (tc $=34$, 448) with 25 degrees of freedom is greater than the tabulated or critical $t(H=1.7081)$ and falls in the rejection zone of the null hypothesis, as well as the value of the critical level $(=0.000)$ is lower than the estimated error $(=0.05)$ then, the null hypothesis is rejected and the research hypothesis is accepted, that is, it is concluded that the debate strategy has produced positive effects in the oral communication skills in children of 5 years of the Initial Educational Institution № 318 Jean Piaget.
\end{abstract}

Keywords: Discussion strategy, oral communicative skills, linguistic, pragmatic, paralanguage, kinésica.

Historial del artículo:

Recibido, 17 de octubre de 2016; aceptado, 13 de mayo de 2017; disponible en línea, 25 de junio de 2017

* Profesora asociada de la Universidad Nacional Intercultural de la Amazonía, Perú.

Correo: atanacia.santacruz@gmail.com 


\section{INTRODUCCIÓN}

El objetivo de la investigación consistió en demostrar los efectos de la estrategia del debate en las competencias comunicativas orales en los niños de 5 años de la Institución Educativa Inicial N $N^{\circ} 318$ Jean Piaget, Yarinacocha en Ucayali, al 2015. Como objetivos específicos se tuvo los siguientes: a) Demostrar los efectos de la estrategia del debate en la dimensión lingüística en los niños de 5 años de la Institución Educativa Inicial $N^{\circ} 318$ Jean Piaget, Yarinacocha-2015; b) Demostrar los efectos de la estrategia del debate en la dimensión pragmática en los niños de 5 años de la Institución Educativa Inicial $N^{\circ} 318$ Jean Piaget - Yarinacocha, 2015; c) Demostrar los efectos de la estrategia del debate en la dimensión paralingüística en los niños de 5 años de la Institución Educativa Inicial $N^{\circ} 318$ Jean Piaget - Yarinacocha, 2015; y, d) Demostrar los efectos de la estrategia del debate en la dimensión kinésica en los niños de 5 años de la Institución Educativa Inicial N ${ }^{\circ} 318$ Jean Piaget, Yarinacocha- 2015.

La investigación se sustenta en el aprendizaje de los niños y niñas han aprendido poner en marcha sus conocimientos, son capaces de manifestar lo que piensan y lo que sienten, reflexionar de manera crítica acerca de la realidad, respetar la opinión de los otros, entre otros. La investigación se realizó con la finalidad de saber convivir con una mente abierta, flexible y crítica. Si se desarrolla estas competencias en los primeros años de la formación académica, se tendrá estudiantes capaces de entender y comprender el mundo, así como futuros ciudadanos más pensantes. Lo anterior es congruente con lo que De Zubiría (2014) expresa: "se debe enseñar a desarrollar competencias transversales para aprender a pensar, convivir, interpretar, leer, escribir y hablar".

\section{MATERIAL Y MÉTODOS}

El tipo de estudio de la presente investigación fue aplicado. Según Hernández, R., Fernández, C. y Baptista, P. (2010). La investigación aplicada resuelve problemas inmediatos en contextos reales con la finalidad de generalizar para futuras investigaciones afines. Y el nivel de investigación es explicativo.

Respecto a las variables de estudio, para la Variable Independiente Estrategias de Debate se ha utilizado papelotes, cartulinas, plumones, cinta maskintape, libros de textos, resúmenes, sesiones de aprendizaje, folletos, materiales diversos del internet, etc.

Para la Variable Dependiente Competencias Comunicativas Orales, se ha utilizado la ficha de observación de entrada y salida, lo cual constó de 16 interrogantes. Se ejecutó en 17 sesiones. El diseño preexperimental con pre y postest, con un grupo. Su esquema según Hernández, et al. (2010) es el siguiente:

\section{GE: $01 \quad X \quad 02$}

Asimismo, la población de estudio estuvo constituida por 26 niños y niñas.

Tabla 1: Resumen de medidas de tendencia central de pretest y postest.

\begin{tabular}{lcrrrr}
\hline \multicolumn{1}{c}{ Valoración } & & \multicolumn{2}{c}{ Pretest } & \multicolumn{2}{c}{ Postest } \\
\hline Cualitativa & Cuantitativa & \multicolumn{1}{c}{$\mathrm{f}$} & \multicolumn{1}{c}{$\%$} & \multicolumn{1}{c}{$\mathrm{f}$} & \multicolumn{1}{c}{$\%$} \\
\hline Inicio & $00-10$ & 26 & $100 \%$ & 0 & $0 \%$ \\
Proceso & $11-13$ & 0 & $0 \%$ & 25 & $96 \%$ \\
Logro previsto & $14-17$ & 0 & $0 \%$ & 1 & $4 \%$ \\
Logro destacado & $18-20$ & 0 & $0 \%$ & 0 & $0 \%$ \\
\hline Total & & 26 & $100 \%$ & 26 & 100 \\
\hline Promedio & & & 6,45 & & 12,28 \\
\hline
\end{tabular}

\section{RESULTADOS}

En el pretest el $100 \%$ de los niños se encontraba con una valoración entre 0 a 10; en el postest el $96 \%$ de los niños se ubicó con una valoración entre 11 a 13 y un $4 \%$ con una valoración de 14 a 17 o tuvo un logro previsto.

Contrastación de la hipótesis general:

Tabla 2: Análisis estadístico.

\begin{tabular}{|c|c|c|c|c|c|c|c|c|}
\hline & \multicolumn{5}{|c|}{ Diferencias emparejadas } & \multirow[b]{3}{*}{$T$} & \multirow[b]{3}{*}{ gl } & \multirow{3}{*}{$\begin{array}{l}\text { Sig. } \\
\text { (bilateral) }\end{array}$} \\
\hline & \multirow[b]{2}{*}{ Media } & \multirow{2}{*}{ 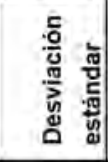 } & \multirow{2}{*}{$\begin{array}{l}\text { Media de } \\
\text { error } \\
\text { estándar } \\
\end{array}$} & \multicolumn{2}{|c|}{$\begin{array}{l}95 \% \text { de intervalo de } \\
\text { confianza de la } \\
\text { diferencia }\end{array}$} & & & \\
\hline & & & & Inferior & Superior & & & \\
\hline $\begin{array}{l}\text { Postest } \\
\text { Pretest }\end{array}$ & 13,5000 & 3,47851 & 0,68219 & 22,09500 & 24,90500 & 34,448 & 25 & 0,000 \\
\hline
\end{tabular}


$\mathrm{H}_{\mathrm{i}}$ : La estrategia del debate produce efectos positivos en las competencias comunicativas orales en los niños de 5 años de la Institución Educativa Inicial N 318 Jean Piaget, Yarinacocha-2015.

$\mathrm{H}_{\mathrm{o}}$ : La estrategia del debate no produce efectos positivos en las competencias comunicativas orales en los niños de 5 años de la Institución Educativa Inicial $N^{\circ} 318$ Jean Piaget, Yarinacocha-2015.

\section{Decisión y conclusión}

Como la $\dagger$ calculada $\left(t_{c}=34,448\right)$ con 25 grados de libertad es mayor que la + tabulada o crítica $\left(t_{t}=\right.$ 1,7081 ) cae en la zona de rechazo de la hipótesis nula, así también el valor del nivel crítico $(=0,000)$ es menor que el error estimado $(=0,05)$ entonces se rechaza la hipótesis nula y se acepta la hipótesis de investigación, es decir: La estrategia del debate produce efectos positivos en las competencias comunicativas orales en los niños de 5 años de la Institución Educativa Inicial N 318 Jean Piaget, Yarinacocha- 2015.

\section{DISCUSIÓN}

Respecto a la hipótesis general como la $t$ calculada $\left(t_{c}\right.$ $=34,448$ ) con 25 grados de libertad es mayor que la $t$ tabulada o crítica $\left(t_{t}=1,7081\right)$ cae en la zona de rechazo de la hipótesis nula, así también el valor del nivel crítico $(=0,000)$ es menor que el error estimado ( $=0.05$ ) entonces se rechaza la hipótesis nula y se acepta la hipótesis de investigación, es decir: La Estrategia del Debate produce efectos positivos en las Competencias Comunicativas Orales en los niños de 5 años de la Institución Educativa Inicial N ${ }^{\circ} 318$ Jean Piaget, Yarinacocha- 2015.

Respecto al resultado en sí, se tiene que Montenegro (2014) en su investigación La Estrategia Mesa Redonda desarrolla las Competencias Comunicativas Orales. Asimismo, Sánchez y Palma, (2014) refuerza la investigación en la medida que la evaluación de las competencias comunicativas contribuye a que los niños muestren mayor seguridad para expresarse de forma oral frente a sus compañeros.

Por otra parte, Marín (2013) fundamentó algo importante en su trabajo, que la secuencia didáctica permite valorar el punto de vista, permite tomar posiciones personales en el desarrollo de competencias argumentativas. Lo que confirma lo hallado en nuestro trabajo.

También Freire y Ong, citado en Sánchez y Brito (2015) coinciden plenamente cuando afirma, es vital que se recobre el valor de la palabra expresada en la competencia oral. Ya que todo vocablo que se exprese pervive una intención, cada palabra tiene poder y cada gesto, ademán o movimiento blinda una acción. Lo cual refuerza nuestro hallazgo en la investigación.

Asimismo, Uribe (2011), insiste en que el impulso de las competencias comunicativas es transversal, puesto que se desarrolla el pensamiento en todas las disciplinas del conocimiento.

\section{Dimensión Lingüística}

La $\dagger_{\text {calculada }}\left(t_{c}=19,670\right)$ con 25 grados de libertad es mayor que la $t$ tabulada o crítica $\left(t_{+}=1,7081\right)$ cae en la zona de rechazo de la hipótesis nula, así también el valor del nivel crítico $(=0,000)$ es menor que el error estimado $(=0,05)$ entonces se rechaza la hipótesis nula y se acepta la hipótesis de investigación; es decir, La Estrategia del Debate produce efectos positivos en la dimensión Lingüística en los niños de 5 años de la Institución Educativa Inicial № 318 Jean Piaget, Yarinacocha-2015.

La investigación de Palma y Castañeda (2014) corroboran la investigación al afirmar, que la simulación de una litigación oral para entrenar a los estudiantes de Derecho, se ha comprobado su efectividad en el desarrollo de competencias argumentativas.

Del mismo modo, De Longhi et al. (2012) afirman que se debe promover discursos coherentes, centrados esencialmente en habilidades cognitivas y lingüísticas, ya que favorece a la comunicación.

Asimismo, Calsamiglia y Tusón (2008) afirman que, el contexto social tiene que ser tomado en cuenta en las interacciones y las interpretaciones, con ello sus características lingüísticas y culturales; reconociendo y valorando en su diversidad de saberes.

De la misma forma, Gadamer (1996) enfatiza en que los seres humanos están inmersos en el lenguaje, en su lengua madre, ellos interpretan sentidos dentro del horizonte de actuación y comprenden. Y Chomsky (1966) afirma que se tiene que toda persona es generadora de diálogo, y la misión de la competencia lingüística es guiar el aprendizaje de la lengua. Lo cual consolida lo hallado en este trabajo.

\section{Dimensión Pragmática}

La $t$ calculada $\left(t_{c}=30,2000\right)$ con 25 grados de libertad es mayor que la $t$ tabulada o crítica $\left(t_{t}=1,7081\right)$ cae en la zona de rechazo de la hipótesis nula, así también el valor del nivel crítico $(=0,000)$ es menor que el error estimado $(=0,05)$ entonces se rechaza la hipótesis nula y se acepta la hipótesis de investigación; es decir, La Estrategia del Debate produce efectos positivos en la dimensión Pragmática en los niños de 5 años de la Institución Educativa Inicial N ${ }^{\circ} 318$ Jean Piaget, Yarinacocha-2015. 
Dichos hallazgos son congruentes con el reporte de Palacino (2007) en la investigación Competencias comunicativas, aprendizaje y enseñanza de las Ciencias naturales, destacando que sí, es apta para incrementar el grado de desarrollo de las competencias comunicativas de tipo discursivo. Palou y Bosch (2005) confirman, que las múltiples situaciones que se produce en el aula para ejercitar la palabra, además de reflejar la dinámica del grupo, son, en potencia situaciones privilegiadas. Lo cual refuerza nuestros hallazgos.

\section{Dimensión Paralenguaje}

La $\dagger$ calculada $\left(\dagger_{c}=21,389\right)$ con 25 grados de libertad

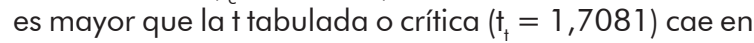
la zona de rechazo de la hipótesis nula, así también el valor del nivel crítico $(=0,000)$ es menor que el error estimado $(=0,05)$ entonces se rechaza la hipótesis nula y se acepta la hipótesis de investigación, es decir: La Estrategia del Debate produce efectos positivos en la dimensión Paralingüística en los niños de 5 años de la Institución Educativa Inicial $N^{\circ} 318$ Jean Piaget, Yarinacocha-2015. La investigación de Cardona y Celis (2011) corroboran nuestra investigación en la medida que las estrategias para mejorar la expresión oral mejoraron satisfactoriamente el proceso en cuanto a la voz, la postura, la argumentación, la mirada entre otros aspectos.

\section{Dimensión Kinésica}

La $t$ calculada $\left(\dagger_{c}=31,801\right)$ con 25 grados de libertad es mayor que la $t$ tabulada o crítica $\left(t_{t}=1.7081\right)$ cae en la zona de rechazo de la hipótesis nula, así también el valor del nivel crítico $(=0,000)$ es menor que el error estimado $(=0,05)$ entonces se rechaza la hipótesis nula y se acepta la hipótesis de investigación, es decir: La estrategia del debate produce efectos positivos en la dimensión Kinésica en los niños de 5 años de la Institución Educativa Inicial No318 Jean Piaget Yarinacocha, 2015. Montenegro (2014) ratifica, que la aplicación de La Estrategia Mesa Redonda desarrolla las competencias comunicativas orales en el nivel Kinésica.

Finalmente, Trejo, Tecuatl, Jiménez y Muriel (2005) aseveran que la expresión corporal está presente en todas las acciones que realizamos. Esa capacidad se conjuga en capacidades para desarrollar la sensibilidad y experimentar el ritmo, los movimientos, la lateralidad y el espacio a través de la utilización del cuerpo, encaminados al desarrollo de la creatividad y comunicación.

\section{Las conclusiones son:}

1. Se ha demostrado con un nivel de significancia del $5 \%$ y una $\left(t_{c}=34,448>t_{t}=1,7081\right)$, que la Estrategia del Debate ha producido efectos positivos en las Competencias Comunicativas Orales en los niños de 5 años de la Institución Educativa Inicial N ${ }^{\circ} 318$ Jean Piaget, Yarinacocha- 2015.

2. Se ha demostrado con un nivel de significancia del $5 \%$ y una $\left(t_{c}=19,670>t_{t}=1,7081\right)$, que la Estrategia del Debate ha producido efectos positivos en la dimensión Lingüística en los niños de 5 años de la Institución Educativa Inicial N ${ }^{\circ} 318$ Jean Piaget, Yarinacocha-, 2015.

3. Se ha demostrado con un nivel de significancia del $5 \%$ y una $\left(t_{c}=30,2000>t_{t}=1,7081\right)$, que la Estrategia del Debate ha producido efectos positivos en la dimensión Pragmática en los niños de 5 años de la Institución Educativa Inicial N 318 Jean Piaget, Yarinacocha-2015.

4. Se ha demostrado con un nivel de significancia del $5 \%$ y una $\left(t_{c}=21,389>t_{t}=1,7081\right)$, que la Estrategia del Debate ha producido efectos positivos en la dimensión Paralingüística en los niños de 5 años de la Institución Educativa Inicial №318 Jean Piaget - Yarinacocha, 2015.

5. Se ha demostrado con un nivel de significancia del $5 \%$ y una $\left(t_{t}=31,801>t_{t}=1,7081\right)$, que la Estrategia del Debate ha producido efectos positivos en la dimensión Kinésica en los niños de 5 años de la Institución Educativa Inicial $N^{\circ} 318$ Jean Piaget - Yarinacocha, 2015.

\section{REFERENCIAS BIBLIOGRÁFICAS}

Calsamiglia, H., \& Tusón, A. (2008). Las cosas del decir. Manual de análisis del discurso. Barcelona: Ariel.

Cardona, J., \& Celis, M. (2011). Estrategias para mejorar la expresión oral en el grado cuarto de educación de básica primaria. Bogotá: Florencia Caquetá. Obtenido de https://edudistancia2001. wikispaces.com/file/view/ESTRATEGIAS+PARA+M EJORAR+LA+EXPRESION+ORAL+EN+EL+GRAD $\mathrm{O}+4 \% \mathrm{C} 2 \% \mathrm{BO}+\mathrm{DE}+\mathrm{BASICA}+\mathrm{PRIMARIA}$. pdf

Chomsky, N. (1970). Aspectos de la Teoría de la sintaxis. Madrid: Aguilar.

De Longui, A., Ferreyra, A., Peme, C., Bermudez, G., Quse, L., Martínez, S., \& et al. (2012). La interacción comunicativa en clases de ciencias naturales. Un análisis didáctico a través de circuitos discursivos. Eureka sobre Enseñanza y Divulgación de las Ciencias, 178-195.

De Zubiría, S. J. (20 de Abril de 2014). Las pruebas PISA żcómo mejorar? Las pruebas PISA żcómo mejorar? Bogotá, no sé, Colombia: Razón Pública. Obtenido de http://www.razonpublica.com/index. 
php/econom-y-sociedad-temas-29/7571-laspruebas-pisa-\%C2\%BFc\%C3\%B3mo-mejorar-losresultados.html; 2014

Gadamer, H. G. (1996). Verdad y método (Vol. 1). Salamanca: Sígueme.

Hernández, R., Fernández, C., \& Baptista, P. (2010). Metodología de la Investigación Científica. Bogotá: Mc Graw Hill.

Marín, G. (2013). Incidencia de una secuencia didáctica, centrada en la lectura crítica de diversos textos y en la discusión oral sobre los mismos, en el desarrollo de competencias argumentativas. Bogotá: municipio de Marsella Risaralda.

Montenegro, H. (2014). Estrategia de mesa redonda para desarrollar competencias comunicativas orales en estudiantes de la Universidad Nacional HermilioValdizán. Huánuco: Universidad Nacional HermilioValdizán.

Palacino, F. (2007). Competencias comunicativas, aprendizaje y enseñanza de las Ciencias Naturales: un enfoque lúdico. Instituto Colombiano para el Fomento de la Educación Superior [ICFES]. Revista Electrónica de Enseñanza de las Ciencias, 6(2), 275-298. Obtenido de http://www.saum.uvigo.es/ reec/volumenes/volumen6/ART4_Vol6_N2

Palma, M., \& Castañeda, M. T. (2014). Litigación oral: estrategia de enseñanza para el desarrollo de competencias argumentativas en la formación de profesores de educación secundaria. Asturias:
Universidad de Oviedo. Obtenido de http:// ac.elscdn.com/S0210277314000274/1 s 2. 0 -S0210277314000274-main.pdf? 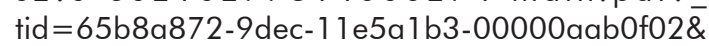 acdnat $=1449607615$ _b8436deeef05e689e337 $1428 c 4 b 7362$

Palou, J., \& Bosch, C. (2005). La lengua oral en la escuela. 10 experiencias didácticas. Barcelona: Graó.

Sánchez, E. J., \& Palma, B. (2014). La evaluación de las competencias comunicativas para el fortalecimiento del lenguaje oral en niños de primer año de preescolar. Bogotá: Jardines de Niños "Blandina Torres de Marín" y "Papalocalli".

Sánchez, J. M., \& Brito, N. E. (2015). Desarrollo de competencias comunicativas mediante la lectura crítica, escritura creativa y expresión oral. Universidad de la Costa-Colombia. Revista Encuentros, Universidad Autónoma del Caribe.

Trejo, O., Tecuatl, D., Jiménez, J., \& Muriel, S. (2005). Educación Creativa: Proyectos escolares. Barcelona: Ediciones Aeroméxico.

Uribe, G. A. (2011). Prácticas de lectura y escritura académicas en la universidad colombiana. Armenia: Universidad del Quindío. Obtenido de http://magisinvestigacioneducacion.javeriana.edu. co 